\title{
Prise en charge de la violence domestique en Suisse romande
}

\section{Jean Martin}

Membre de la rédaction

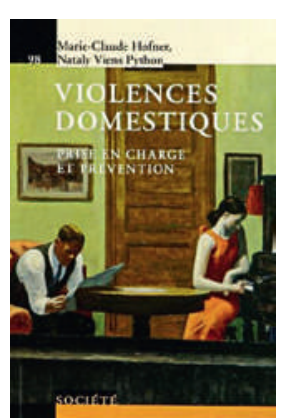

Marie-Claude Hofner, Nataly Viens Python Violences domestiques - Prise en charge et prévention

Lausanne: Presses Polytechniques et Universitaires Romandes, collection «Le Savoir suisse»; 2014.

134 pages. $17.50 \mathrm{CHF}$

ISBN 978-2-889-15052-6

La maltraitance est aujourd'hui un sujet majeur de santé publique et sociale. Longtemps, ni les médecins ni les responsables au sein de la localité ou de la société ne se sont trop préoccupés de la violence domestique. Héritage du droit de vie et de mort du pater familias romain, on admettait que le chef de famille était libre d'agir comme il l'entendait dans son propre foyer. Notion aussi que le rôle de la loi et de l'Etat "s'arrête sur le seuil de la maison". L'idée que des violences soient exercées, parfois comme une occurrence quotidienne, sur des êtres dépendants souvent fragiles (enfants, femmes - cas échéant enceintes, personnes âgées), était gênante, inconciliable avec l'assistance et l'affection qu'on se doit au sein d'une famille. A l'époque de mes études dans les années 1960 , on découvrait la notion d'enfant battu, parfois avec scepticisme en dépit des études pionnières de Kempe et Silverman aux USA. Voyant de petits enfants arriver à l'hôpital avec contusions, fractures ou brûlures, la première réaction n'était pas d'imaginer que les parents puissent en être responsables. Cela ne veut pas dire que, au cours des siècles, personne n'observait les effets de tant de mauvais traitements ni ne se doutait de leur origine (voir de grands auteurs comme Dickens, Hugo, Zola); mais, pour des raisons de principe de l'époque ou de crainte d'être intrusif dans la vie privée des autres, on préférait ne pas se mêler «de ce qui ne nous regarde pas».

\section{Prise de conscience - un problème aux multiples origines}

Les institutions et professionnels médico-sociaux ont progressivement émergé de cette sorte de cécité qu'elle soit inconsciente ou «préférée». Dans les der- nières décennies du $\mathrm{XX}^{\mathrm{e}}$ siècle, le médecin cantonal que j'étais a été plus fréquemment interpelé par des professionnels médico-sociaux à propos de maltraitance. Dans le canton de Vaud, il convient de mentionner l'engagement alors de la pédopsychiatre Odette Masson, qui a donné lieu à la mise sur pied d'une Commission cantonale de prévention des mauvais traitements. D’autres se sont préoccupés du problème, notamment le service d'urgences du CHUV, partenaire majeur de l'histoire décrite par les deux auteures de "Violences domestiques». Marie-Claude Hofner est médecin de santé publique, elle a été active de longue date en médecine sociale et préventive et est associée à l'Unité de médecine des violences du CHUV. Nataly Viens Python est une infirmière québécoise qui enseigne à la Haute Ecole La Source, à Lausanne.

Extraits: «Ces hommes et ces femmes (battus) ont été en contact avec leur médecin, leur gynécologue, le médecin de l'entreprise, d'autres intervenants encore, et aucun de ces professionnels ne leur a posé les questions qui auraient permis de susciter une confidence, de demander un conseil [...] En le faisant, ces soignants ne dépasseraient-ils pas les limites de leur fonction? Une patiente dira: 'J'aurais tant voulu qu'on me demande, en tête-à-tête, si j'avais une autre version des faits s.»

«L’incompréhension du phénomène, les préjugés, l'ignorance des professionnels sont autant de circonstances qui poussent les victimes à dissimuler les souffrances vécues. Elles mènent en quelque sorte une double vie. Il y a celle de l'extérieur, construite pour donner le change et maintenir une image conforme du couple et de la famille, et celle de l'intérieur, faite de peur, de vigilance permanente, de crises.» «Les différents types de violence ne sont pas des phénomènes isolés et étanches. La violence physique ou sexuelle est toujours accompagnée de violence psychologique. L'influence de l'alcool est évidente, aussi bien au sein de la famille qu'en dehors d'elle. La précarité économique est un facteur de risque.» Se souvenir du rôle possible d'une dépendance et de la trahison d'une confiance: «L'OMS insiste sur les liens entre l'auteur et la victime: la maltraitance de l'enfant entraîne un préjudice pour sa santé, sa survie, son déve- 
loppement ou sa dignité dans le contexte d'une relation de responsabilité, de confiance ou de pouvoir.»

\section{Programme "C'est assez", puis Unité de médecine des violences}

Depuis 200o, un programme de prévention de la violence conjugale, intitulé "C'est assez», est mis en place à Lausanne, par le Bureau cantonal de l'égalité, le Centre des urgences du CHUV et l'Institut de médecine sociale et préventive. $\mathrm{Vu}$ l'importance des aspects médico-légaux, est créée en 2005 l'Unité de médecine des violences (UMV), rattachée au Centre universitaire romand de médecine légale. Ouverture d'une consultation hospitalière en 2006, au sein de laquelle des infirmières spécifiquement formées œuvrent en collaboration avec les médecins légistes.

L'«aventure» illustrée dans le livre est exemplaire d'une réflexion et action de santé publique. Les auteures se réfèrent à un «modèle écologique de la violence», soutenu par l'OMS, avec quatre niveaux étroitement imbriqués: individuel, relationnel, communautaire et sociétal. Démarche prenant en compte les circonstances des personnes et de leur milieu proche et plus large. Développant des actions multisectorielles avec les personnes et services concernés: professionnels des soins et du social, policiers, instances judiciaires etc. Créant des réseaux pour répondre aux besoins des personnes maltraitées et disséminant des "cartes du réseau»: cartes permettant, quel que soit son lieu propre d'observation et de travail, de se mettre sans délai en rapport avec ceux dont, dans d'autres domaines, les compétences et moyens sont nécessaires au traitement immédiat et à la gestion ultérieure d'une maltraitance.

Dans les mesures de réseautage, on trouve entretiens, visites sur le terrain (y compris journées et nuits passées avec des policiers), journées de rencontre et formation; collaboration avec de "grands partenaires» comme le Centre d'accueil Malley-Prairie, à Lausanne. Traitement de problèmes juridiques comme celui, pas rare, de l'épouse battue d'origine étrangère qui risque de perdre son permis $\mathrm{B}$ si elle quitte le domicile conjugal et d'être alors renvoyée.

Même si un tel programme est à l'évidence indispensable, les auteures relèvent avec modestie: «En matière de violence, les travaux scientifiques comme les expériences pratiques mettent en lumière que les mesures à prendre (du point de vue notamment de la prévention) ne sont pas pour l'essentiel du domaine de la santé mais de la responsabilité de la communauté, des décideurs politiques et économiques, et des citoyens.» Affirmation d'un axiome de santé pu- blique. Les activités de l'UMV font toutefois face à certains obstacles: «L'interdisciplinarité de l'équipe et la volonté de mener le projet en collaboration étroite avec des secteurs non académiques rendaient difficile son intégration dans une structure universitaire.»

\section{Quelques dimensions encore}

Remarque critique à propos d'échos médiatiques: «L'impact sur la communauté se fait également sentir par l'utilisation faite de la ‘violence` dans les médias et les débats publics. Tout est mis sous la même appellation; incivilités urbaines, littering, délinquances des jeunes. Ces amalgames donnent une vision tronquée de la réalité, mettant en avant les évènements spectaculaires, sans précaution quant à leur interprétation. Ceci n'informe guère, donne à s'émouvoir et bien peu à penser, ne propose ni analyse ni solution et maintient la confusion.»

A propos des enfants victimes de ces situations: "Aujourd'hui, tant l'expérience des professionnels que les recherches à large échelle confirment que les enfants qui assistent à des actes de violence conjugale dans leur famille présentent les mêmes problèmes de santé et les mêmes difficultés de développement que les enfants qui ont subi des maltraitances qui leur étaient directement destinées.»

Un élément d'importance, dans le sens de l'évolution actuelle de partenariat avec les patients, de leur 〈empowerment»: «Si les besoins ont été identifiés, les personnes concernées - victimes et auteurs - n'ont pas encore été intégrées dans l'élaboration des programmes [...]. L'expérience des femmes et des hommes qui se sont émancipés de relations violentes, leur appréciation des messages et des formes d'aide proposées doivent désormais enrichir les programmes [...] Pour les auteurs de violences eux-mêmes, la violence est une impasse, fait souffrir, rétrécit le champ des possibles." Il importe d'offrir aux personnes violentes des programmes leur permettant de sortir de l'isolement, de prendre conscience des impacts de leurs comportements et d'élaborer des alternatives à la violence.

En conclusion, lecture recommandée, tant par l'importance du problème traité que par la présentation de la mise en œuvre d'une stratégie interdisciplinaire et multisectorielle [1].

1 Sur ce thème, récemment dans le BMS: Hofner MC, Rodondi PY. Violence domestique -5 points essentiels pour la prise en charge au cabinet. Bull Méd Suisses. 2015;96(7):243-4.

Romann C. La violence domestique: un sujet qui concerne aussi la médecine. Bull Méd Suisses. 2015;96(7):213.

Tschudin S. Prävention und Bekämpfung von häuslicher Gewalt. Bull Méd Suisses. 2015; 96(7):242-4. 